\title{
Dynamic simulation of three-dimensional motion of the human arm and upper body
}

\author{
C. Y. A. Chan $^{1}$, A. B. Thornton-Trump ${ }^{1}$ \\ \& K. M. A. Weiss-Bundy ${ }^{2}$ \\ ${ }^{I}$ Department of Mechanical and Manufacturing Engineering, \\ University of Manitoba, Winnipeg, Canada \\ ${ }^{2}$ Department of Mechanical Engineering, University of Calgary, \\ Calgary, Canada
}

\begin{abstract}
This paper presents the use of trajectory following methods for determining the motion of the human upper body segments as they relate to arm segments and human back movements in three-dimensional space. Given a hand trajectory in global space and the position of the base of the spine, inverse dynamics can be applied to develop the total moment at each joint of a linkage representing the human arm and back. Using the co-ordinate systems and kinematics developed in previous works, a fully scalable simulation of the human upper body is developed and the dynamic and static moments at each joint are predicted for a worker performing a repetitive task. The joint moments are predicted for a simulated worker and then compared to those calculated for a human subject performing the same task. The joint moment curves are compared for three different workstation geometries for both the simulation and the human subject.

Keywords: dynamics, human back, human upper arm, three-dimensional simulation, Euler equation, anatomical scaling.
\end{abstract}

\section{Introduction}

Repetitive strain injuries to workers in industry who are required to use inappropriate workstation geometries have a large impact on society. Industry has medical and training costs as well as a loss of production resulting from repetitive strain injuries. In addition to the loss of income and quality of life to the worker, there are additional medical costs. A tool to simulate the motion of a 
worker and workstation in virtual space and predict the joint moments at the shoulder and the lower back will be useful for workstation design. A method of synthesizing the motion of the trajectory of a work piece as it is moved by a worker has been determined by Chan et al. [1]. In order to predict the joint moments, a nine degree-of-freedom dynamic simulation program is required. The program must include all the effects of mass distribution, linear accelerations and angular accelerations of the work object and body segments as the object is moved through space. There are many quasi-static programs for analysis of human subject motion [2,3], but few which simulate the motion of the human arm and upper body without using motion data acquired from a human subject.

The simulation of the motion of a worker performing a task requires information on the mass and mass moments of inertia of the human arm segments. The worker and the workstation must be scalable in a virtual space environment in order to simulate the worker and the workstation of various sizes. The motion of all the arm and back segments must be related to the constraints of the human anatomy in such a way that muscle origins and insertions can be defined in terms of bone anatomy so that muscle forces and moment, and moment arms can be made available. These co-ordinate problems have been previously addressed [1]. However, the aspects of scalability, bone anatomy and mass distribution were not addressed.

The objectives of this paper are to develop a nine degree-of-freedom dynamic model integrated with mass distribution data such that human subjects can be scaled. Using the trajectory- following method, an inverse-dynamic model can be developed to determine accelerations and joint moments. By changing the workstation geometry it can be shown that joint moments change significantly with workstation geometry and task time.

\section{Methodology}

The establishment of co-ordinate systems and co-ordinate transformation matrices were given in Chan et al. [1]. Using a parabola as a hypothetical work object trajectory for the task, the motion of the arm and upper body segments through space are defined by solving for body segment positions at each position along the work object trajectory. Redundancy in the possible body segment geometries is eliminated by selecting joint positions compatible with ergonomic angles. Where such angles are not possible due to work object collisions with other objects in the workstation environment, the angles nearest to the ergonomic values are used.

A human scaling methodology was developed using the data from HumanScale [4] and defining three body types: ectomorphic, mesomorphic, and endomorphic [6]. The subject or worker was considered to be thin or large if his/her body mass index was twenty percent less than or greater than the mean. By choosing the height and weight of the subject the mean mass and the mass moments of inertia of each body segment were made available to the dynamic program. Mathematical functions which determined the acceleration, constant 
speed and deceleration portions of the trajectory were presented by Chan et. al. $[1]$.

In order to determine the joint moments at the back, the shoulder, the elbow and the wrist, Euler equations of motion were used to determine the threedimensional motion of each segment. Euler equations of motion include Newton's second law and the equations of angular motion [5]. Equation (1) is the general equation of angular motion in matrix form. The motion of each body segment was calculated relative to its own local co-ordinate and then transformed to the adjacent co-ordinate and then to the fixed reference coordinate.

$$
\begin{aligned}
{\left[\begin{array}{l}
\mathbf{M}_{x} \\
\mathbf{M}_{y} \\
\mathbf{M}_{z}
\end{array}\right]=} & {\left[\begin{array}{ccc}
I_{x x} & -I_{x y} & -I_{x z} \\
-I_{y x} & I_{y y} & -I_{y z} \\
-I_{z x} & -I_{z y} & I_{z z}
\end{array}\right]\left[\begin{array}{l}
\alpha_{x} \\
\alpha_{y} \\
\alpha_{z}
\end{array}\right] } \\
& +\left[\begin{array}{ccc}
0 & -\omega_{x} & \omega_{y} \\
\omega_{z} & 0 & -\omega_{x} \\
-\omega_{y} & \omega_{x} & 0
\end{array}\right]\left[\begin{array}{ccc}
I_{x x} & -I_{x y} & -I_{x z} \\
-I_{y x} & I_{y y} & -I_{y z} \\
-I_{z x} & -I_{z y} & I_{z z}
\end{array}\right]\left[\begin{array}{l}
\omega_{x} \\
\omega_{y} \\
\omega_{z}
\end{array}\right]
\end{aligned}
$$

It must be noted that the angular velocity and angular acceleration of each angle rotation is calculated by differentiating the angle of rotation with respect to time across the full task time. In order to develop the motion of each segment in terms of the Eulerian angles, the angular velocity and angular acceleration of each segment must be represented in terms of the rate of change of the rotational angle associated with each segment. It is also important to note that the angular velocity and angular acceleration terms in the Euler equations of motion are the absolute angular velocity and the absolute angular acceleration of each body segment.

The free body diagram of the forearm is shown in Figure 1 and the equations for the calculation of the joint moments at the elbow are shown in equations (2) and (3).

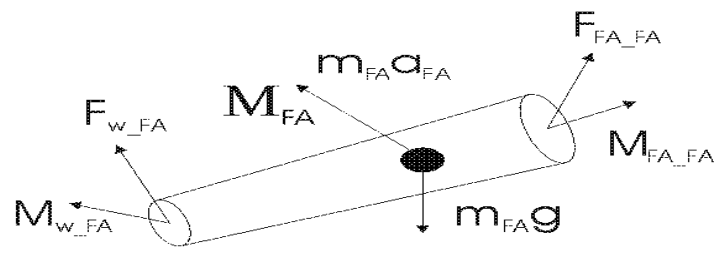

Figure 1: Free body diagram of the forearm.

In Figure 1, $\mathrm{F}_{\mathrm{w}_{-} \mathrm{FA}}$ represents the total force applied by the motion of the hand and object at the wrist in the forearm co-ordinate, $\mathrm{M}_{\mathrm{w}_{-} \mathrm{FA}}$ the total moment applied by the motion of the hand and object at the wrist in the forearm coordinate, $\mathbf{M}_{\mathbf{F A}}$ the angular moment of the forearm, $\mathrm{F}_{\mathrm{FA} \_\mathrm{FA}}$ the total force of the 
forearm in the forearm co-ordinate, $\mathrm{M}_{\mathrm{FA} F \mathrm{FA}}$ the total moment of the forearm in the forearm co-ordinate, $a_{\mathrm{abs} \text { FA }}$ the absolute angular acceleration of the forearm in the forearm co-ordinate, $a_{\mathrm{FA}}$ the linear acceleration of the forearm, $\mathrm{g}_{\mathrm{FA}}$ the gravity in the forearm co-ordinate. The acceleration terms indicate the true three-dimensional dynamic properties of the program. The free body diagram and the calculation of the joint moments of the other segments are similar to those of the forearm.

$$
\begin{aligned}
& \sum F=\left[F_{F A_{-} F A}\right]+m_{F A}\left[g_{F A}\right]+m_{F A}\left[a_{a b s-F A}\right]-\left[F_{w_{-} F A}\right]=0 \\
& {\left[F_{F A \_F A}\right]=-\left(m_{F A}\left[g_{F A}\right]+m_{F A}\left[a_{a b s_{-} F A}\right]-\left[F_{w_{-} F A}\right]\right)} \\
& \sum M=\left[M_{F A_{-} F A}\right]+\left[\begin{array}{c}
-l_{F A} \\
0 \\
0
\end{array}\right] \times m_{F A}\left[g_{F A}\right]+\left[\begin{array}{c}
-l_{F A} \\
0 \\
0
\end{array}\right] \times m_{F A}\left[a_{F A}\right] \\
& +\left[\begin{array}{c}
- \text { Link2 } \\
0 \\
0
\end{array}\right] \times\left[-F_{w_{-} F A}\right]+\left[\mathbf{M}_{F A}\right]-\left[M_{w_{-} F A}\right]=0 \\
& {\left[M_{F A_{-} F A}\right]=-\left(\left[\begin{array}{c}
-l_{F A} \\
0 \\
0
\end{array}\right] \times m_{F A}\left[g_{F A}\right]+\left[\begin{array}{c}
-l_{F A} \\
0 \\
0
\end{array}\right] \times m_{F A}\left[a_{F A}\right]\right.} \\
& \left.+\left[\begin{array}{c}
-\operatorname{Link} 2 \\
0 \\
0
\end{array}\right] \times\left[-F_{w_{-} F A}\right]+\left[\mathbf{M}_{F A}\right]-\left[M_{w_{-} F A}\right]\right)
\end{aligned}
$$

\section{Experimental protocol}

There is no way to measure the moments in the back and the shoulder. Even an in vivo measurement of individual muscle forces cannot be used to determine the moments until an appropriate biomechanical model exists. The predicted joint moments from the simulation program must therefore be generated from some geometry that allows analysis by other means. Such a geometry is created by extending the arm in the coronal anatomical plane, then flexing the forearm through 180 degrees and then back to 0 degrees. The static moments are easily calculated and can be compared to the moments predicted by the dynamic program for very long task times (i.e. slow motion) as shown in Figure 2. Such a test is considered to be quasistatic and the results were in total agreement. Using the same geometry, but a very short task time (i.e. fast motion), the effects of accelerations could be seen in the moment curves of Figure 3 . The test was then repeated with the arm extended in the sagittal plane. The moment curves for slow motion are shown in Figure 4, while those for fast motion in Figure 5. This latter test indicated that the program was working properly in three-dimensional space. 


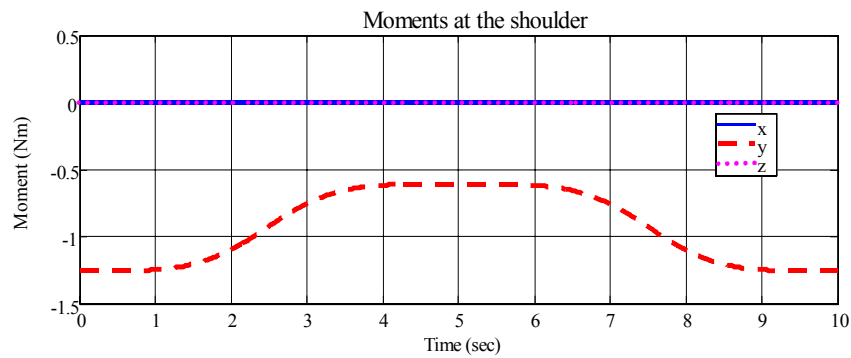

Figure 2: The shoulder moments for the slow motion (coronal anatomical plane).

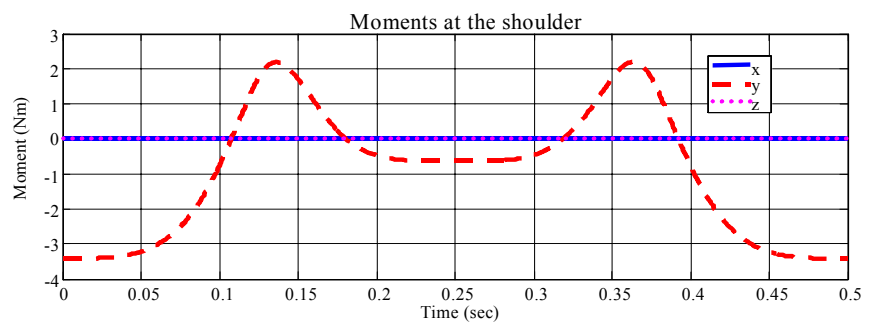

Figure 3: The shoulder moments for the fast motion (coronal anatomical plane).

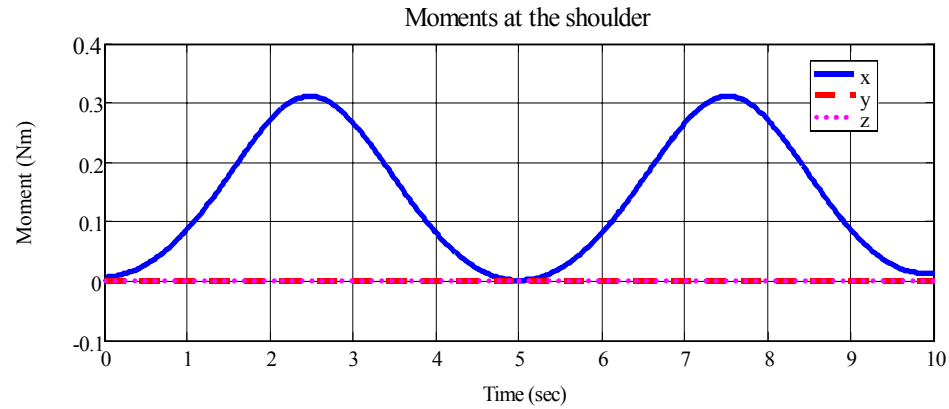

Figure 4: The shoulder moments for the slow motion (sagittal anatomical plane).

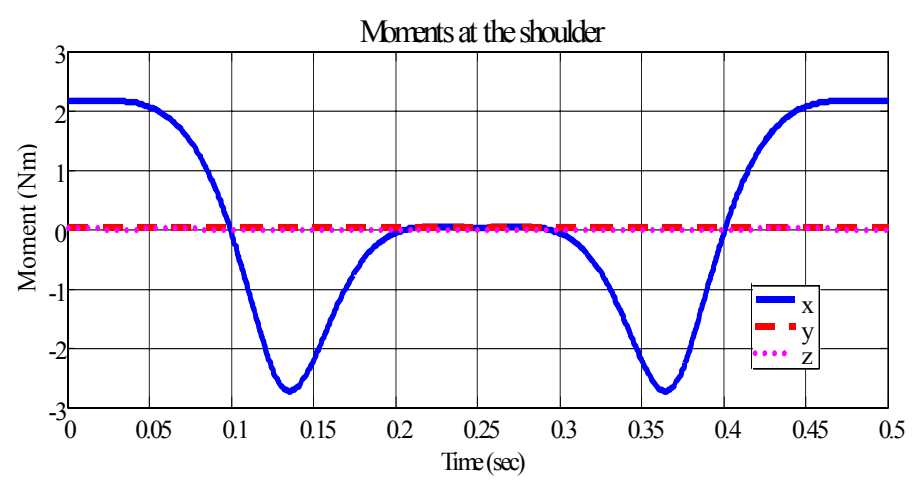

Figure 5: The shoulder moments for the fast motion (sagittal anatomical plane). 
Finally, the task of placing a one-litre bottle into a box sitting on a counter top was then analyzed. The same subject repeated the action six times for each of three different counter heights. The data acquired by four infrared CCD cameras was entered into the dynamic program in order to compare the joint moments at the shoulder generated by the parabolic assumption of the trajectory to the joint moments predicted from human subject data.

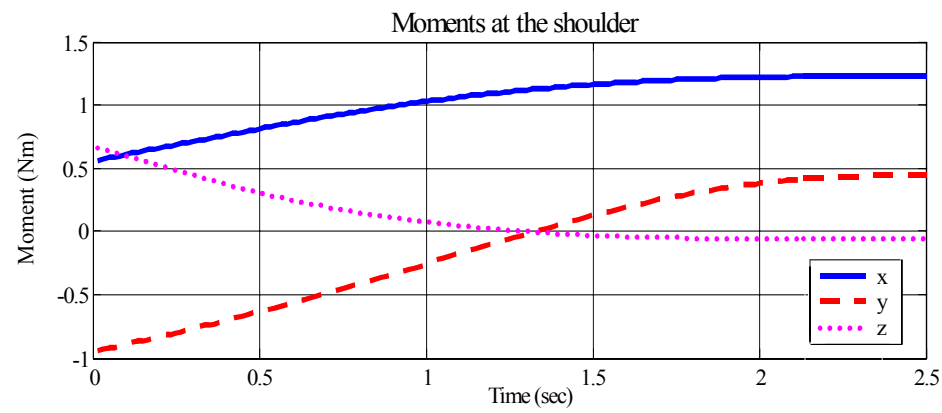

(a)

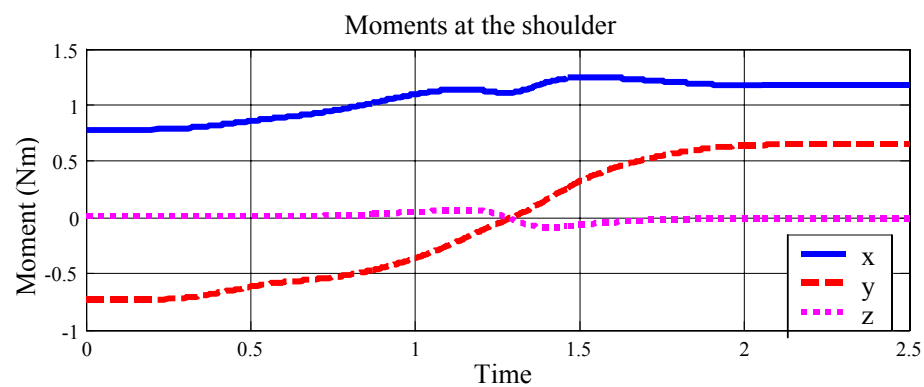

(b)

Figure 6: Shoulder moments predicted using the trajectory from (a) the simulation and (b) the human subject.

\section{Discussion}

Figure 6a shows the joint moments for the medium counter height predictions from the dynamic program, using a parabolic trajectory. Figure $6 \mathrm{~b}$ shows the moments at the shoulder generated by using the trajectory data taken from the human subject. It is noted that the moments predicted by the simulation program are lower in magnitude and considerably smoother over the task than are the moments from the human subject. Since the simulation identifies the origin and target of the work object in space and the trajectory is considered to be known, no feedback is required to place the object on the target. For the human subject, considerable spatial distance judgment is required to place the work object correctly on the target. The human subject thus varies acceleration and deceleration along the trajectory due to feedback information from the eyes. 
Those changes in acceleration create peaks and valleys on the shoulder moment curve. It is possible that sufficient training would reduce the variations such that the human subject could learn to place the object without visual feedback, leading to a smoother moment curve. Such a study was beyond the scope of this work. However, the curves do show that task training is likely very important in avoiding injury.

In a further simulation, a 5-foot tall male worker and an average height counter (30 inches) are used in the simulation program. Figures $7 \mathrm{a}$ and $\mathrm{b}$ show that the maximum shoulder moment changes significantly with the speed at which the worker performs the task. From Figures $7 \mathrm{~b}$ and $\mathrm{c}$, it is seen that the maximum shoulder moment changes significantly with counter height. In other words, industries that place small workers at high counters are more likely to have a high rate of repetitive strain injury.

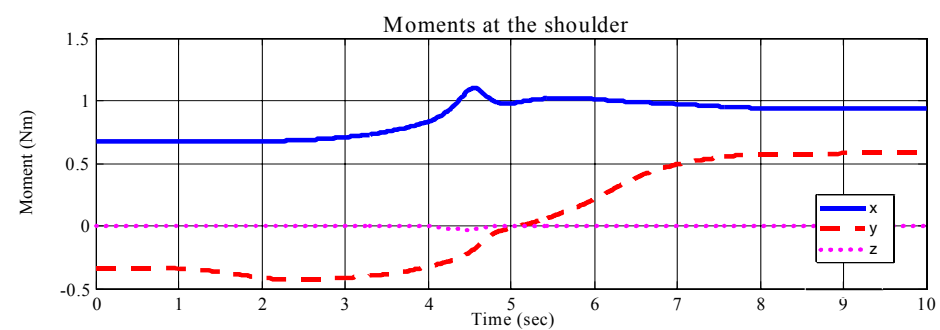

(a)

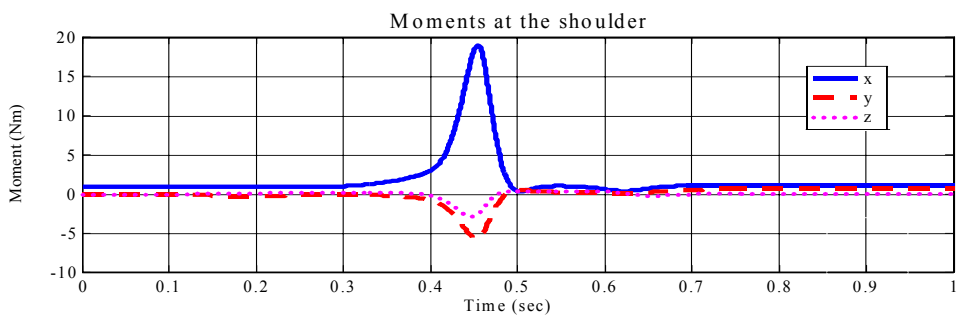

(b)

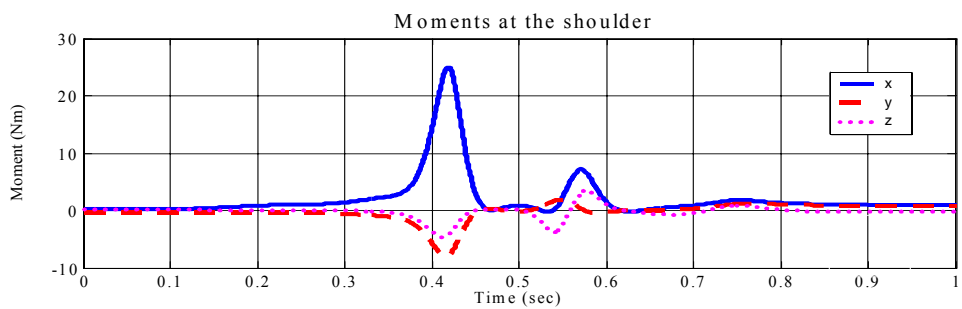

(c)

Figure 7: Shoulder moments simulated for (a) the slow motion and (b) the fast motion using the average counter. (c) Shoulder moments simulated for the fast motion using the high counter. 
Finally, a simulation using a counter with a section cut down to allow the top of the box to be at counter height shows a very large reduction in shoulder moment, as seen in Figure 8. All of these observations are consistent with workstation evolvement in industry.

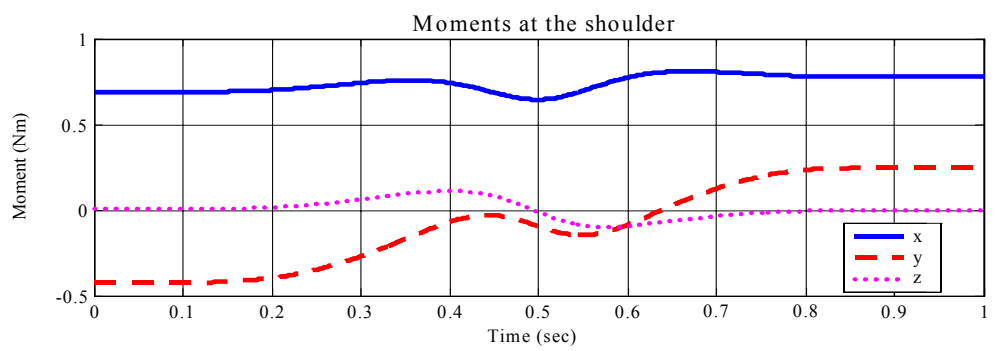

Figure 8: Shoulder moments simulated using a counter with a section cut down and a parabolic trajectory.

When the worker stands far away from the workstation, in order to reach the work object and follow the work object trajectory, back rotations are employed. A simulation is done using arm extension first, and then the upper body rotates about three rotation axes to add the reach. During the simulation the back rotation mechanism is activated only when the object is outside the reaching distance of the fully extended arm. Figures 9 and 10 show that the moments at the L5 increased significantly as the back rotations are employed. The risk of repetitive strain injury is higher if the workstation is not properly designed thus forcing the worker to reach out to perform the task.

Finally, a simulation using a very low counter with a height of 0.3 meters shows that the worker has to bend down to pick up the work object and move it to the target position. It can be seen in Figure 11 that the maximum back moment changes significantly with a very low counter. In other words, tall workers working at low counters also have a high risk of repetitive strain injury.

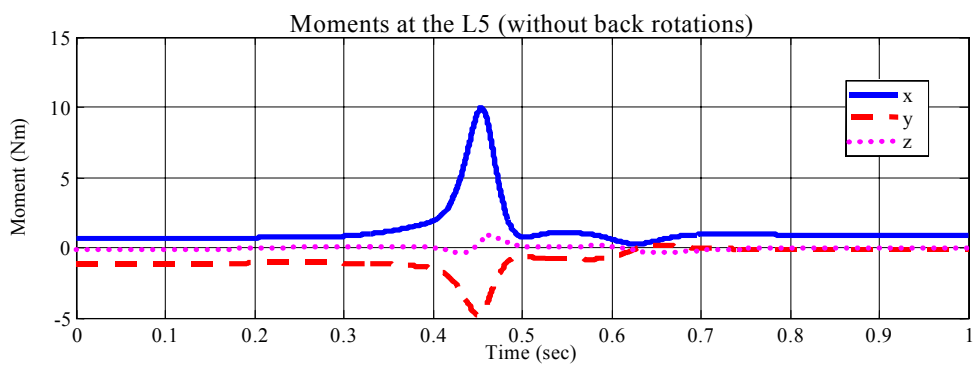

Figure 9: Moments at L5 with no back rotation employed, parabolic trajectory (worker stands $0.3 \mathrm{~m}$ away from the object). 


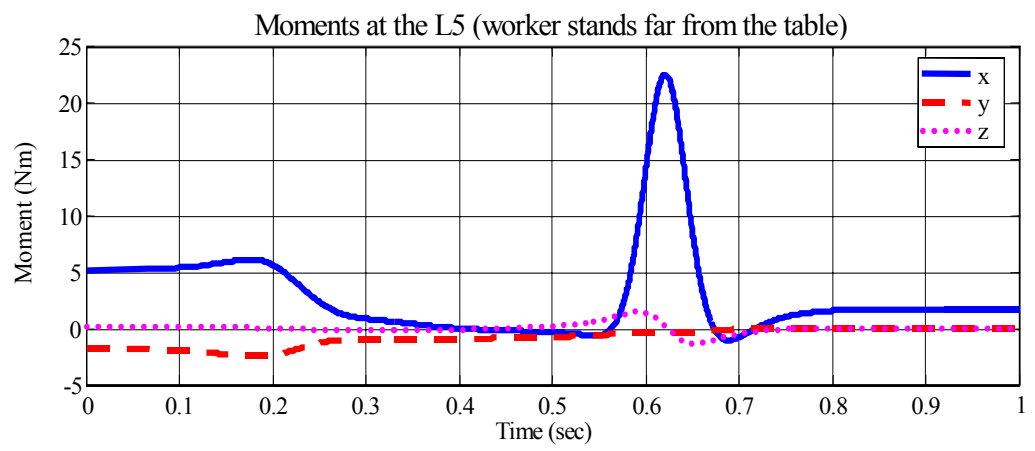

Figure 10: Moments at L5 with back rotations, parabolic trajectory (worker stands $0.6 \mathrm{~m}$ away from the object).

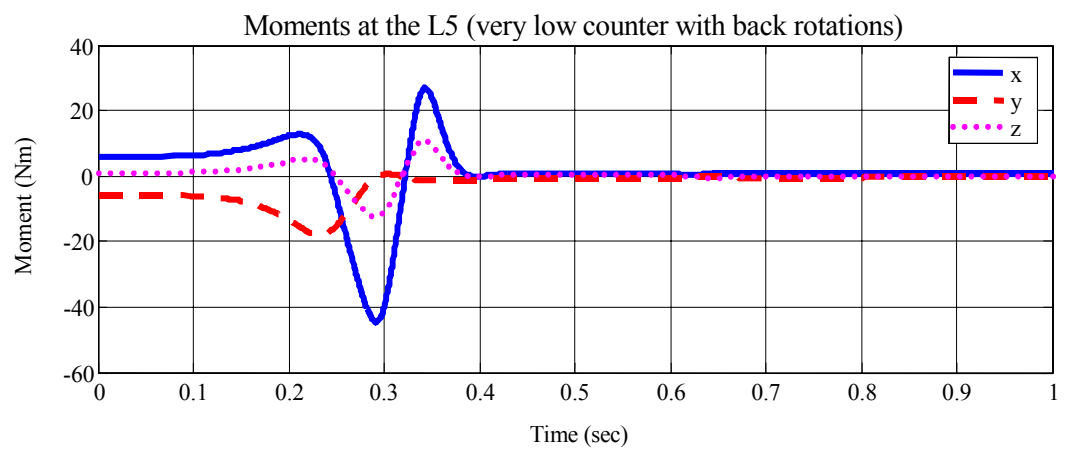

Figure 11: Moments at L5 (with a $0.3 \mathrm{~m}$ high counter).

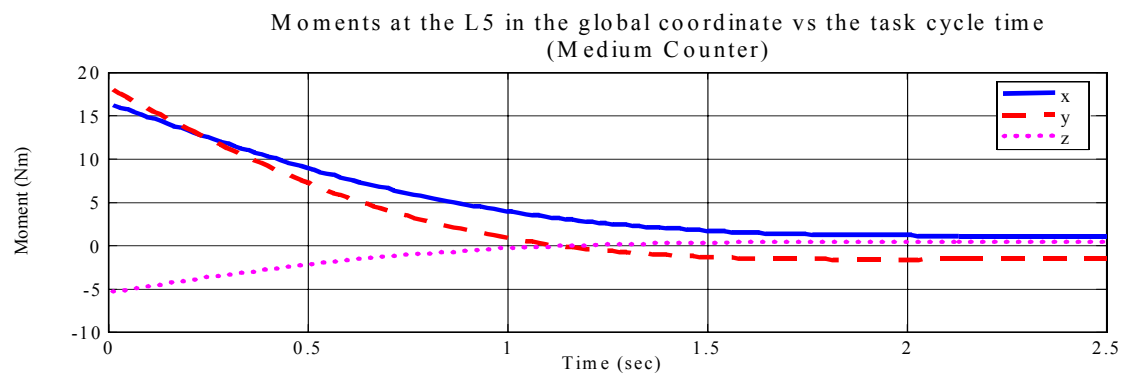

Figure 12: Moments at L5 with back rotations at a medium counter height predicted using the trajectory data from the human subject.

Figure 12 shows the effect on the joint moment at L5 of leaning forward and following the work object trajectory extracted from a human subject. The trajectory contains variable acceleration with an oscillation period of 0.4 seconds. The oscillation in the acceleration is possibly developed from the need 
of a human subject to judge the distance from the target and to control the speed of movement. Simulation results from the parabolic trajectory do not contain the acceleration oscillations since no feedback mechanism is built into the simulation program. The moment variations are thus seen to cause much larger peak moments at L5 than the simulation would predict. With the need to lean forward, the increased moments at L5 indicate that back muscle fatigue and possible strain injury risk would be much larger than workstation designs that allow a more upright posture.

An improvement to the simulation program would be the addition of an acceleration program routine to allow the acceleration oscillation effects to be included. Further data acquisition would be required to characterize the oscillations in relation to the task and required object trajectory.

From the above simulation results, it can be concluded that the dynamic program developed is sensitive enough to give good guidance in workstation design. While the predicted moments from the simulation are lower than those of a human subject, more knowledge of the effects of visual feedback in targeting could develop acceleration functions to be used in the simulation to improve the magnitude of the simulation predictions.

\section{Conclusions}

Using a trajectory following approach, a three-dimensional program predicts the dynamic effects of task time on back, shoulder, elbow and wrist moments. The program is capable of simulating the dynamics of a task with the scaleable worker and workstation in a virtual space environment, thus eliminating the need to build, test and rebuild workstations. The simulation is sensitive to workstation geometry changes and can quantify the relative effects on shoulder moment as a function of workstation geometry changes.

Angles at the joints of limb segments can also be extracted from the program. The program can thus give guidance concerning the ergonomic joint angles actually used in a task at a specific workstation.

\section{References}

[1] C.Y.A. Chan, A.B. Thornton-Trump, K.M.A. Weiss-Bundy, Modelling the human upper body in three-dimensional motion, Proceedings of Design and Nature II, Rhodes 2004, (WIT Press, Southampton, 2004) 451-460

[2] R. Fazel-Rezai, E. Shwedyk, S. Onyshko, and J.E. Cooper (1996), Power Analysis of Upperlimb Movement, Proceedings of 18th Annual International Conference of the IEEE Engineering in Medicine and Biology-Proceedings, 2, 621-622.

[3] X. Zhang, D.B. Chaffin, and D. Thompsho (1997), Development of dynamic simulation models of seated reaching motions while driving, $S A E$ Special Publications Progress with Human Factors in Automotive Design: Seating Comfort, Visibility, and Safety, 1242, 101-105. 
[4] N. Diffrient, A. R. Tilley, and J. C. Bardagjy, Humanscale 1/2/3 Manual, (The MIT Press, Massachusetts, 1990).

[5] A. Bedford and W. Fowler, Engineering Mechanics - Dynamics, (Addison-Wesley Publishing Company, Inc., Massachusetts, 1995).

[6] K. Weiss-Bundy (2002), Determination of Dynamic Muscle Forces Across the Elbow using Three-Dimensional Vector Modelling, M.Sc. Thesis, Department of Mechanical Engineering, University of Manitoba. 